\title{
Automatic Multi-pumping Flow System for the Chemiluminometric Screening of Scavenging Capacity against Singlet Oxygen
}

\author{
Ana F. T. Silva, Karine L. Marques, ${ }^{\dagger}$ João L. M. Santos, and José L. F. C. Lima \\ REQUIMTE, Departamento de Química, Faculdade de Farmácia, Universidade do Porto, Rua Aníbal Cunha, \\ 164, 4099-030, Porto, Portugal
}

\begin{abstract}
An automated multi-pumping flow system was developed for the in-line generation of singlet oxygen $\left({ }^{1} \mathrm{O}_{2}\right)$ and subsequent assessment of the scavenging capacity against this reactive species. ${ }^{1} \mathrm{O}_{2}$ was generated by dismutation of hydrogen peroxide catalyzed by molybdate ions. The evaluation of the scavenging capacity was based on the inhibition of the chemiluminescence reaction of luminol with ${ }^{1} \mathrm{O}_{2}$. The proposed system used solenoid micro-pumps as the only active components of the flow manifold, enabling the reproducible insertion and efficient mixing of sample and reagents as well as the transportation of the sample zone towards detection for chemiluminescence measurement, assuring a strictly reproducible timing of all analytical tasks. Several compounds were evaluated as possible ${ }^{1} \mathrm{O}_{2}$ scavengers. The obtained results showed that only ascorbic acid, dipyrone and tryptophan exhibited scavenging capacity, with $\mathrm{IC}_{50}$ values of $3.36 \times 10^{-5}, 7.84 \times 10^{-5}$ and $1.28 \times 10^{-2} \mathrm{~mol} \mathrm{~L}^{-1}$, respectively.
\end{abstract}

(Received April 6, 2011; Accepted June 1, 2011; Published August 10, 2011)

\section{Introduction}

Singlet oxygen $\left({ }^{1} \mathrm{O}_{2}\right)$ is a highly unstable and reactive oxygen species (ROS) with large significance in many chemical and biological processes. Although playing an important role in cellular metabolism as regulator in signaling processes and in host defense against intruding microorganisms, ${ }^{1} \mathrm{O}_{2}$ also exhibits toxic effects to the organism when overproduced. In fact, ${ }^{1} \mathrm{O}_{2}$ may be harmful due to its ability to react with DNA, lipids and proteins inhibiting their normal function, and such changes are involved in the development of cancer, cardiovascular diseases and aging process. ${ }^{1-3}$

For this reason, the development of in vitro screening methodologies to assess the scavenging effects of biologically active compounds and therapeutic drugs against ${ }^{1} \mathrm{O}_{2}$ is extremely important. In this context, several methods for evaluation of reactivity towards ${ }^{1} \mathrm{O}_{2}$, have been reported, based on electron spin resonance $(\mathrm{ESR})^{4,5}$ and absorbance, ${ }^{6,7}$ fluorescence, ${ }^{8,9}$ direct near-infrared luminescence $\mathrm{e}^{10,11}$ and chemiluminescence $(\mathrm{CL})^{12,13}$ measurements. Although ESR can detect free radicals specifically and directly, its sensitivity is relatively low and it requires specialized and expensive equipment. The spectrophotometric assay is commonly used because of its simplicity; however, the sensitivity is much lower when compared to other methods. Fluorescence is a sensitive technique and new fluorescent probes are being developed in order to increase selectivity. Methodologies based on the characteristic luminescence emission of ${ }^{1} \mathrm{O}_{2}$ in the near-infrared spectral region at $1270 \mathrm{~nm}$ are very specific, but its use is sometimes difficult because the intensity is extremely weak Alternatively, CL methods not requiring excitation light sources

$\dagger$ To whom correspondence should be addressed.

E-mail: karine.marques@ff.up.pt have been widely used because of their high sensitivity and the use of inexpensive instruments.

Most of the reported methods are based on manual discrete procedures and exploit the ability of the different compounds to inhibit the oxidation reactions mediated by ${ }^{1} \mathrm{O}_{2}$. Considering that ${ }^{1} \mathrm{O}_{2}$ is highly unstable and presents a very short lifetime ${ }^{14}$ that makes it difficult to detect, these batch procedures are susceptible to operational errors such as inadequate sample/reagent mixing that could affect precision and accuracy. Moreover, the redox processes that led ultimately to light emission could involve distinct short-lived species, which renders the reproducible timing of the measurements under non-equilibrium conditions a critical issue. In addition, batch methods are time-consuming, laborious and costly, so they are not suitable for routine or screening analysis.

Automation of these methodologies can help to circumvent these limitations, particularly resorting to continuous flow techniques. ${ }^{15}$ In this context, sequential injection analysis has been applied to the CL detection of antioxidant activity against ${ }^{1} \mathrm{O}_{2}$ which was enzymatically produced using lactoperoxidase enzyme. ${ }^{13}$

Multi-pumping flow systems (MPFS) are an excellent tool for automation since they allow the implementation of fast, low-cost and reliable analytical procedures. ${ }^{16}$ MPFS are characterized by a high versatility and simplicity due to the use of only one type of active component, solenoid micro-pumps, that are multi-task devices responsible for solutions insertion, propelling and commutation. When compared to other flow techniques, MPFS exhibit an increased mixing capacity provided by the pulsed flowing streams resultant from the micro-pumps actuation. This high mixing potential is a valuable feature because it provides improved sample/reagent intermixing that facilitates reaction development which is particularly attractive when carrying out measurements that involve short-lived species such as singlet oxygen. ${ }^{17}$ 
Furthermore, MPFS are computer-controlled systems offering a reproducible mixing of solutions, excellent precision on timing events and more flexibility concerning the method development and operation since any changes in the parameters are accomplished using software control. ${ }^{18}$

One of the main purposes of this work is to study the reactivity of several compounds towards ${ }^{1} \mathrm{O}_{2}$ and evaluate their possible scavenging capacity. To this end, a second goal was set: the development of an automated multi-pumping flow system for the implementation of in-line generation of ${ }^{1} \mathrm{O}_{2}$ and the assessment of scavenging capacity against ${ }^{1} \mathrm{O}_{2}$ with CL detection.

In vitro, ${ }^{1} \mathrm{O}_{2}$ can be generated through photochemical reactions, ${ }^{4}$ enzymatic methods ${ }^{7,13}$ and chemical reactions. ${ }^{8,9,11,12}$ In this work, ${ }^{1} \mathrm{O}_{2}$ was generated through catalytic dismutation of hydrogen peroxide by $\mathrm{MoO}_{4}{ }^{2-}$. Hydrogen peroxide spontaneous dismutation into water and ${ }^{1} \mathrm{O}_{2}$ has an extremely low rate, but is efficiently catalyzed by $\mathrm{MoO}_{4}{ }^{-2} \cdot{ }^{19}$

The high versatility and operational simplicity of the developed MPFS allowed the implementation of a methodology for evaluation of the scavenging activity based on the inhibitory effect on the CL reaction of luminol with ${ }^{1} \mathrm{O}_{2}$ that was applied to the compounds: sodium azide, mannitol, glutathione, tryptophan, ascorbic acid, dipyrone and nifedipine.

\section{Experimental}

\section{Reagents and chemicals}

All chemicals were of analytical reagent grade and doubly deionized water (specific conductivity $<0.1 \mu \mathrm{S} \mathrm{cm}^{-1}$ ) was used as a carrier and in solution preparations. Hydrogen peroxide $30 \%(\mathrm{v} / \mathrm{v})$, ascorbic acid and sodium carbonate were purchased from Merck (Darmstadt, Germany). Luminol (5-amino-2,3dihydro-phthalazinedione), sodium azide, dipyrone, glutathione and nifedipine were purchased from Sigma-Aldrich (St. Louis, MO). Sodium molybdate and mannitol were purchased from Riedel-de-Haen (Seelze, Germany). Tryptophan was purchased from Fluka (Buchs, Switzerland).

A $3.5 \times 10^{-2} \mathrm{~mol} \mathrm{~L}^{-1}$ luminol stock solution was prepared by dissolving $310.8 \mathrm{mg}$ of luminol in $50 \mathrm{~mL}$ of carbonate buffer $0.1 \mathrm{~mol} \mathrm{~L}^{-1}, \mathrm{pH}$ 11.5. This solution was protected from light and kept in a refrigerator. A working solution of $2.5 \times 10^{-3} \mathrm{~mol} \mathrm{~L}^{-1}$ luminol was daily prepared by dilution of the stock solution with carbonate buffer.

A $2.5 \times 10^{-2} \mathrm{~mol} \mathrm{~L}^{-1} \mathrm{H}_{2} \mathrm{O}_{2}$ solution was daily prepared by rigorous dilution of a $30 \%(\mathrm{v} / \mathrm{v})$ stock solution with deionized water. A sodium molybdate $5 \times 10^{-2} \mathrm{~mol} \mathrm{~L}^{-1}$ solution was prepared by dissolving $302 \mathrm{mg}$ of $\mathrm{Na}_{2} \mathrm{MoO}_{4} \cdot 2 \mathrm{H}_{2} \mathrm{O}$ in $25 \mathrm{~mL}$ of deionized water.

Stock solutions of sodium azide, tryptophan, mannitol, dipyrone, glutathione, and ascorbic acid were daily prepared by dissolving the required amount in deionized water. Nifedipine stock solution was prepared in ethanol. All calibration solutions were prepared by dilution of stock solution in water, except nifedipine calibration solutions that were prepared by dilution of the stock solution in $10 \%$ ethanol.

\section{Equipment}

For chemiluminescence measurements, an FP2020Plus Model (Jasco, Easton, USA) chemiluminescence detector was used. The detector included a modular flow-cell consisting of a spiraled $0.8 \mathrm{~mm}$ i.d. PTFE tube with an internal volume of $100 \mu \mathrm{L}$ that was positioned in front of a highly sensitive end-on photomultiplier. As active components of the developed flow

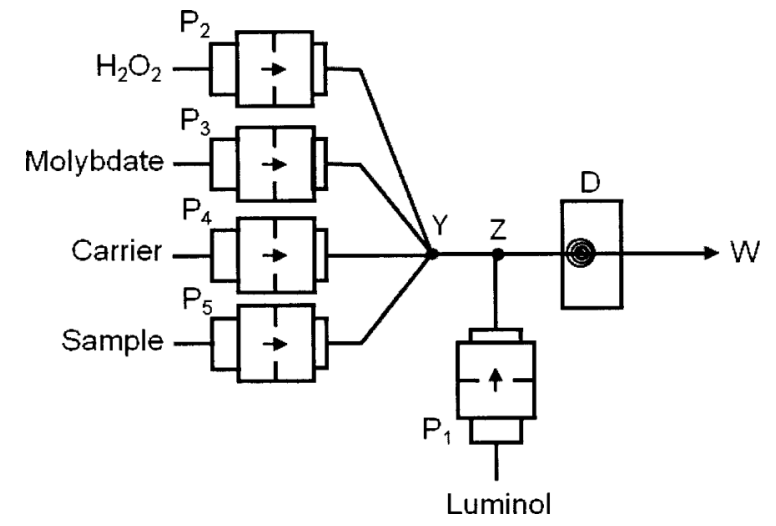

Fig. 1 Flow manifold diagram. $\mathrm{P}_{1}, \mathrm{P}_{2}, \mathrm{P}_{3}, \mathrm{P}_{4}$ and $\mathrm{P}_{5}$, solenoid micro-pumps; Y and $\mathrm{Z}$, confluence points; $\mathrm{D}$, detector; $\mathrm{W}$, waste; carrier, deionized water.

manifold, multiple solenoid actuated micro-pumps Ref. 120SP (BIOCHEM Inc., Boonton, USA), were used, these were connected by using flow lines and reaction coils made of 0.8 $\mathrm{mm}$ i.d. PTFE tubing. The micro-pumps were of the fixed displacement diaphragm type, and they were solenoid operated and dispensed $10 \mu \mathrm{L}$ per stroke. Home-made end-fittings, connectors and confluence points were also used.

A Julabo Variomag EC thermostatized water bath (Julabo Labor Technik GmbH, Germany) was used to maintain the solutions at $50^{\circ} \mathrm{C}$.

A CoolDrive ${ }^{\mathrm{TM}}$ circuit (NResearch, Caldwell, USA) was used as a power drive to operate the solenoid micro-pumps. Data acquisition and control of the analytical system were accomplished with a computer through a PC-LABCard Model PCL-711B interface card from Advantech (Taiwan). The software was developed in QuickBASIC 4.5.

\section{Flow manifold}

The analytical flow manifold (Fig. 1) comprised five solenoid-actuated micro-pumps responsible for the individual management of solutions and was designed to enable the implementation of ${ }^{1} \mathrm{O}_{2}$ in-line generation and to perform the assay for evaluation of the tested compounds reactivity towards ${ }^{1} \mathrm{O}_{2}$.

The analytical cycle started with the simultaneous activation of the solenoid micro-pumps $\mathrm{P}_{2}$ and $\mathrm{P}_{5}$, alternating with $\mathrm{P}_{1}$ and $\mathrm{P}_{3}$, for insertion of $\mathrm{H}_{2} \mathrm{O}_{2}$ and sample or luminol and $\mathrm{MoO}_{4}{ }^{2-}$ solutions, respectively. This way, $\mathrm{H}_{2} \mathrm{O}_{2}$ and sample solutions were simultaneously introduced into the analytical path and intercalated with small aliquots of $\mathrm{MoO}_{4}{ }^{2-}$ solution at confluence point $\mathrm{Y}$. Consequently, ${ }^{1} \mathrm{O}_{2}$ was generated by reaction with the tested compounds. At the same time, luminol solution was inserted at confluence point $\mathrm{Z}$, merging with the previously formed reaction zone and reacting with the remaining ${ }^{1} \mathrm{O}_{2}$. Finally, solenoid micro-pump $\mathrm{P}_{4}$ was activated to carry the reaction zone to the detector, yielding the analytical signal.

In the proposed flow manifold, solutions mixing, reaction zone establishment and development was accomplished by two complementary processes: insertion of solutions by binary sampling strategy and the pulsed flow nature of the flowing stream. The chaotic movement of the solution in all directions produced by the micro-pumps actuation contributes to an efficient homogenization of the sample zone and thus favors reaction development. 


\section{Results and Discussion}

One of the objectives of the present work was the development of an automatic methodology for evaluation of the reactivity of selected compounds towards ${ }^{1} \mathrm{O}_{2}$. Therefore, an automatic MPFS was conceived, taking into consideration the in situ generation of ${ }^{1} \mathrm{O}_{2}$ by $\mathrm{H}_{2} \mathrm{O}_{2}$ dismutation catalyzed by $\mathrm{MoO}_{4}{ }^{2-}$, the short lifetime of this reactive species and the assessment of the interaction of the tested compounds by monitoring luminol oxidation. Depending on whether the tested compound was a scavenger/antioxidant or a pro-oxidant, its effect in luminol oxidation resulted in the inhibition of CL emission or in an increase of CL emission.

During system optimization, the effects of chemical and instrumental parameters affecting the CL intensity were evaluated. The main purpose was to establish conditions that enabled an analytical signal with a maximum intensity, which would correspond to maximum production of ${ }^{1} \mathrm{O}_{2}$. In addition, possible interference from luminol oxidation by $\mathrm{H}_{2} \mathrm{O}_{2}$ was also evaluated by performing all the experiments without $\mathrm{MoO}_{4}{ }^{2-}$, by inserting water in micro-pump $\mathrm{P}_{3}$.

\section{Flow system optimization}

The great versatility characteristic of MPFS depends on the individual control of each solenoid micro-pump, so that solutions are inserted in the analytical system only when necessary, allowing the synchronization of reagents addition to a precise sample portion and establishing a well-defined reaction zone. The order by which solutions should be inserted into the analytical path was investigated to find the sequence that favored ${ }^{1} \mathrm{O}_{2}$ formation, its reaction with the selected compound and its detection by reaction with luminol. Considering that luminol can be oxidized either by $\mathrm{H}_{2} \mathrm{O}_{2}$ or ${ }^{1} \mathrm{O}_{2}$, the flow system was designed to assure that ${ }^{1} \mathrm{O}_{2}$ was generated prior to the mixing with luminol. This way, $\mathrm{H}_{2} \mathrm{O}_{2}, \mathrm{MoO}_{4}{ }^{2-}$ and sample solutions were simultaneously introduced, thus generating ${ }^{1} \mathrm{O}_{2}$ which would react with the compound under study; subsequently, in a confluence point closer to the detector, luminol was added to the reaction zone previously formed.

An important parameter in ${ }^{1} \mathrm{O}_{2}$ generation, that is relatively slow, ${ }^{20}$ is the time of reaction between $\mathrm{H}_{2} \mathrm{O}_{2}$ and $\mathrm{MoO}_{4}{ }^{2-}$. The presence of a reactor between confluence points $\mathrm{Y}$ and $\mathrm{Z}$ (Fig. 1) would increase the time of reaction between $\mathrm{H}_{2} \mathrm{O}_{2}$ and $\mathrm{MoO}_{4}{ }^{2-}$ leading to a higher production of ${ }^{1} \mathrm{O}_{2}$ and consequently to an increase in the emission intensity. On the other hand, since ${ }^{1} \mathrm{O}_{2}$ presents a reduced lifetime in aqueous medium, ${ }^{14}$ a decrease in $\mathrm{CL}$ emission could occur due to the fact that when the reaction zone formed by $\mathrm{H}_{2} \mathrm{O}_{2}-\mathrm{MoO}_{4}{ }^{2-}$ reached the confluence point $\mathrm{Z}$ there would be no ${ }^{1} \mathrm{O}_{2}$ available to react with luminol. Therefore, the influence of a reactor in the CL emission was evaluated by varying the connection between the two confluence points with reactors of 5,25 and $50 \mathrm{~cm}$. By increasing the length of the reactor, we found that there was a decrease of about $20 \%$ in the radiation emitted in the presence of $\mathrm{MoO}_{4}{ }^{2-}$. These results indicate that the highest amount of ${ }^{1} \mathrm{O}_{2}$ was detected without a reaction coil, using only tubing with the minimum length of $5 \mathrm{~cm}$ to connect the two confluence points.

Another essential parameter in terms of the intensity of the analytical signal is the volume of sample and reagents inserted by solenoid micro-pumps. In MPFS sample and reagents, volumes are defined by the stroke volume and the number of pulses of micro-pump actuation; thus, this volume is always a multiple of stroke volume, $10 \mu \mathrm{L} \times n$ pulses. The effect of sample volume was evaluated in the range of 20 to $120 \mu \mathrm{L}$, that corresponds to a number of pulses from 2 to 12 . It was verified that the analytical signal showed a 3.6 fold increase with the increase of the sample volume from 20 to $60 \mu \mathrm{L}$ and then approached stabilization. As a result, $60 \mu \mathrm{L}$ was the volume of sample used for subsequent studies.

In CL reactions implemented in flow systems, flow rate is a crucial parameter: it influences the time of reaction as well as the residence time of the reaction zone inside the flow cell. The flow rate was evaluated within 0.375 and $1.5 \mathrm{~mL} \mathrm{~min}^{-1}$. We verified that if we increased the flow rate the analytical signal decreased to half the initial value. The highest production of ${ }^{1} \mathrm{O}_{2}$ was obtained for a flow rate of $0.375 \mathrm{~mL} \mathrm{~min}{ }^{-1}$. However, the use of such a reduced flow would result in a very low sampling rate. Therefore a flow rate of $0.5 \mathrm{~mL} \mathrm{~min}{ }^{-1}$ per each micro-pump was chosen, since it corresponds to a compromise between fast determinations without significant reduction in ${ }^{1} \mathrm{O}_{2}$ production. Using this flow rate we started to measure the analytical signal about $3 \mathrm{~s}$ after the beginning of the reaction.

Since luminol is the emitting species, optimization of luminol concentration is very important. Concentrations of luminol ranging from $1 \times 10^{-4}$ to $5 \times 10^{-3} \mathrm{~mol} \mathrm{~L}^{-1}$ were tested. An increase in the analytical signal was verified for concentrations up to $2.5 \times 10^{-3} \mathrm{~mol} \mathrm{~L}^{-1}$, stabilizing for higher concentrations of luminol. When the influence of luminol concentration was evaluated without $\mathrm{MoO}_{4}{ }^{2-}$, the analytical signal slightly increased up to $5 \times 10^{-3} \mathrm{~mol} \mathrm{~L}^{-1}$; nevertheless, the signal was still much lower than the one obtained with $\mathrm{MoO}_{4}{ }^{2-}$. For subsequent work, a luminol concentration of $2.5 \times 10^{-3} \mathrm{~mol} \mathrm{~L}^{-1}$ was chosen.

As previously mentioned, the spontaneous dismutation of $\mathrm{H}_{2} \mathrm{O}_{2}$ presents an extremely low rate, being efficiently catalyzed by $\mathrm{MoO}_{4}{ }^{2-}$ with the formation of ${ }^{1} \mathrm{O}_{2}$. The effect of $\mathrm{MoO}_{4}{ }^{2-}$ on the intensity of the radiation emitted was evaluated for concentrations ranging from $1 \times 10^{-3}$ to $1 \times 10^{-1} \mathrm{~mol} \mathrm{~L}^{-1}$. The production of ${ }^{1} \mathrm{O}_{2}$ increased with the increase in $\mathrm{MoO}_{4}{ }^{2-}$ concentration, reaching its maximum at $5 \times 10^{-2} \mathrm{~mol} \mathrm{~L}^{-1}$, and stabilized for higher concentrations of $\mathrm{MoO}_{4}{ }^{2-}$.

The production of ${ }^{1} \mathrm{O}_{2}$ is directly dependent on the $\mathrm{H}_{2} \mathrm{O}_{2}$ concentration. According to Aubry et al., two molecules of $\mathrm{H}_{2} \mathrm{O}_{2}$ produce one molecule of ${ }^{1} \mathrm{O}_{2} \cdot{ }^{19}$ In this context, $\mathrm{H}_{2} \mathrm{O}_{2}$ concentrations ranging from $5 \times 10^{-4}$ to $5 \times 10^{-2} \mathrm{~mol} \mathrm{~L}^{-1}$ were studied; a maximum emission which corresponded to the higher production of ${ }^{1} \mathrm{O}_{2}$, was obtained for a concentration of $2.5 \times 10^{-2} \mathrm{~mol} \mathrm{~L}^{-1}$. This concentration was used for the following assays.

${ }^{1} \mathrm{O}_{2}$ generation and luminol oxidation are significantly affected by $\mathrm{pH}$, so the optimization of this parameter is required. The rate of ${ }^{1} \mathrm{O}_{2}$ generation showed a maximum at $\mathrm{pH}$ 10.5. ${ }^{19}$ Thus the effect of $\mathrm{pH}$ was investigated using carbonate buffer $10^{-1} \mathrm{~mol} \mathrm{~L}^{-1}$ with $\mathrm{pH} 10.5$ as carrier instead of water; the obtained analytical signal was similar to the one obtained with water. Kawatani et al. reported an analogous result that was attributed to the fact that the mixed solution of $\mathrm{H}_{2} \mathrm{O}_{2}-\mathrm{MoO}_{4}{ }^{2-}$ has a $\mathrm{pH}$ of about $10 .{ }^{21}$ Thus, we continued to use water as carrier. Concerning luminol oxidation, different luminol solutions prepared in $10^{-1} \mathrm{~mol} \mathrm{~L}^{-1}$ carbonate buffer with the $\mathrm{pH}$ adjusted between 9 and 12.5 were evaluated. The results showed that the highest generation of ${ }^{1} \mathrm{O}_{2}$ occurred at $\mathrm{pH} 11.5$, so this value was selected for further work.

The extent of the reactions involved in the determination, their rates and efficiency are temperature-dependent. The effect of temperature was evaluated for $24,30,40,50$ and $60^{\circ} \mathrm{C}$, while the assays were performed using a thermostatized bath to keep solutions at the required temperature. By increasing the temperature, we observed an increase in CL emitted, 


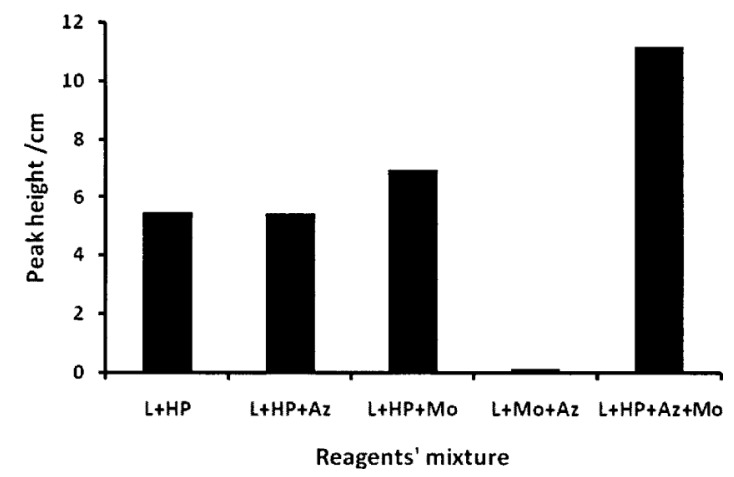

Fig. 2 Analytical signal obtained from different mixtures of the reagents involved in the CL reaction. L, Luminol; HP, hydrogen peroxide; Az, sodium azide; Mo, sodium molybdate.

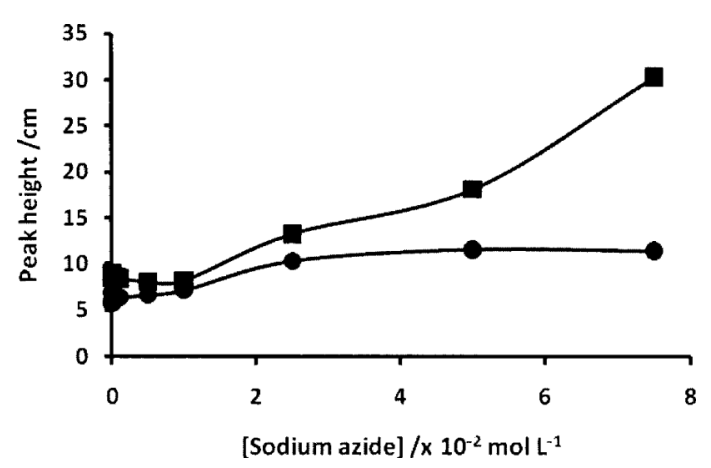

Fig. 3 The effect of sodium azide on the analytical signal. The results refer to the analysis with $\mathrm{MoO}_{4}{ }^{2-}(\mathbf{\square})$ and without $\mathrm{MoO}_{4}{ }^{2-}(\boldsymbol{Q})$.

corresponding to an increase in ${ }^{1} \mathrm{O}_{2}$ production as well as an increase of the $\mathrm{CL}$ reaction rate. When the experiments were performed at $60^{\circ} \mathrm{C}$, there was an increase in the formation of air bubbles; these affected the CL detection, producing irreproducible results. Thus, the assays were performed at $50^{\circ} \mathrm{C}$, representing a compromise that gave the highest analytical signals without formation of air bubbles.

Evaluation of the scavenging capacity against ${ }^{1} \mathrm{O}_{2}$

The main purpose of this work was to investigate the behavior of several compounds towards the reactive species ${ }^{1} \mathrm{O}_{2}$. In this regard, after optimization of the developed MPFS, the reactivity of each selected compound was characterized by variations in the intensity of the CL emitted in the presence of such a compound.

Sodium azide, known to be a specific scavenger for ${ }^{1} \mathrm{O}_{2}$, was tested; instead of the expected decrease in the CL emission, an enhancing effect was observed. For that reason, the source of the CL was investigated by assaying different mixtures of the reagents involved; the results are shown in Fig. 2. First, a significant $\mathrm{CL}$ signal was obtained from the oxidation of luminol by $\mathrm{H}_{2} \mathrm{O}_{2}$. In the second situation, sodium azide was mixed with luminol and $\mathrm{H}_{2} \mathrm{O}_{2}$ and the obtained signal was similar to the one obtained from the first mixture; such a result demonstrates that sodium azide does not interfere in the luminol oxidation by $\mathrm{H}_{2} \mathrm{O}_{2}$. In the third assay, $\mathrm{MoO}_{4}{ }^{2-}$ was added to the mixture of luminol and $\mathrm{H}_{2} \mathrm{O}_{2}$, resulting in an increase in the $\mathrm{CL}$ emitted caused by the formation of ${ }^{1} \mathrm{O}_{2}$. Subsequently, the mixture of luminol with $\mathrm{MoO}_{4}{ }^{2-}$ and sodium azide was assayed, resulting in

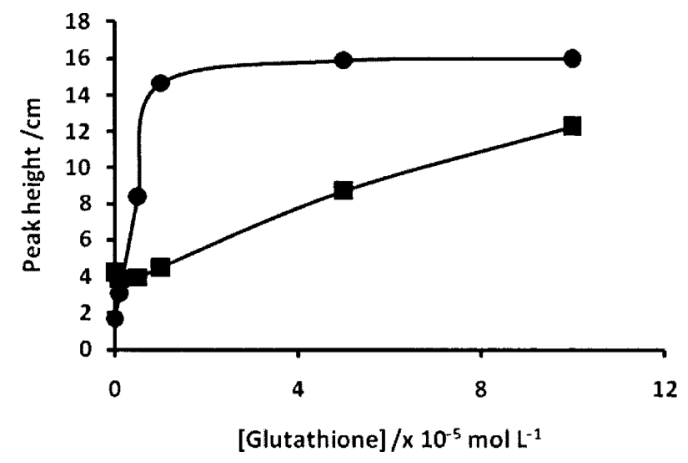

Fig. 4 The effect of glutathione on the analytical signal. The results refer to the analysis with $\mathrm{MoO}_{4}{ }^{2-}(\boldsymbol{\square})$ and without $\mathrm{MoO}_{4}{ }^{2-}$

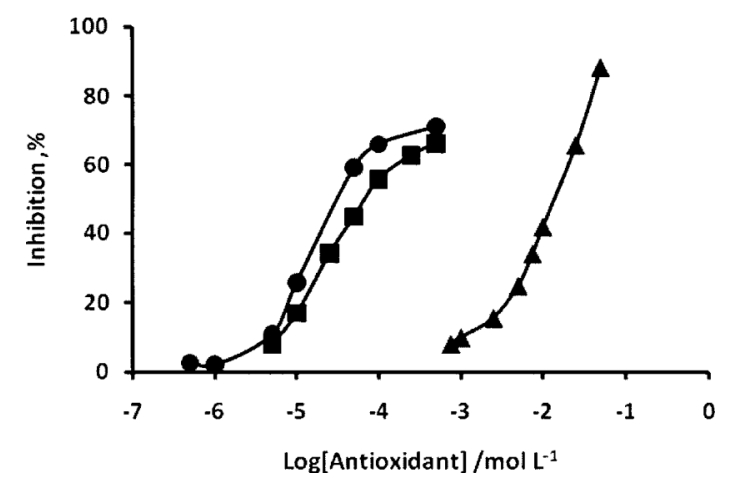

Fig. 5 Inhibition of ${ }^{1} \mathrm{O}_{2}$-luminol CL by ascorbic acid (), dipyrone (ם) and tryptophan (A).

a residual CL emission. Finally, the mixture of luminol, $\mathrm{H}_{2} \mathrm{O}_{2}$, $\mathrm{MoO}_{4}{ }^{2-}$ and sodium azide led to an even higher CL signal, indicating that the emission was the result of the reaction of ${ }^{1} \mathrm{O}_{2}$ with sodium azide.

Afterwards, the effect of sodium azide concentrations ranging from $1 \times 10^{-5}$ to $7.5 \times 10^{-2} \mathrm{~mol} \mathrm{~L}^{-1}$ was evaluated; an increase in the CL emitted was verified by increasing the concentration of sodium azide in the presence of $\mathrm{MoO}_{4}{ }^{2-}$ (Fig. 3). When the assay was performed in the absence of $\mathrm{MoO}_{4}{ }^{2-}$, there was a slight increase in the CL up to $2.5 \times 10^{-2} \mathrm{~mol} \mathrm{~L}^{-1}$ and then the value stabilized. It is known that sodium azide reacts with ${ }^{1} \mathrm{O}_{2}$ with a high rate constant and that the azidyl radical is a strong one-electron oxidant that is able to oxidize thiols and other sulfur compounds, phenols and other products. ${ }^{22}$ Thus, if the reaction rate with sodium azide is higher than that with luminol, azide competes with luminol for ${ }^{1} \mathrm{O}_{2}$, producing azidyl radical which could then oxidize luminol, leading to the increase in CL. Opposite to our results, in the SIA methodology developed for the assessment of antioxidant activity against ${ }^{1} \mathrm{O}_{2}$ with $\mathrm{CL}$ detection, sodium azide lead to a decrease in the radiation emitted. ${ }^{13}$ This discrepancy may be explained by the different experimental conditions, namely the $\mathrm{pH}$. In the SIA methodology a $\mathrm{pH}$ of 4.5 was used, while in our work we used alkaline $\mathrm{pH}$, which could result in a different behavior of sodium azide. In fact, it has been reported that some compounds either inhibit or enhance the luminol CL, depending on the $\mathrm{pH}$ of the solution. ${ }^{23}$ With an increase in $\mathrm{pH}$, inhibition for most compounds tended to convert toward enhancement. Furthermore, in the SIA methodology luminol was dissolved in dimethylformamide and sodium azide was dissolved in 
Table 1 Scavenging capacity against ${ }^{1} \mathrm{O}_{2}$ using the multi-pumping flow system developed

\begin{tabular}{cccc}
\hline Tested compound & Inhibition curve & $r^{\mathrm{a}}$ & $\mathrm{IC}_{50^{\mathrm{b}}}$ \\
\hline Tryptophan & $I \%=63.20 \log C+168.87$ & 0.997 & $1.28 \times 10^{-2} \pm 0.06 \times 10^{-2}$ \\
Dipyrone & $I \%=34.05 \log C+189.15$ & 0.998 & $7.84 \times 10^{-5} \pm 0.49 \times 10^{-5}$ \\
Ascorbic acid & $I \%=43.75 \log C+243.93$ & 0.99 & $3.36 \times 10^{-5} \pm 0.47 \times 10^{-5}$ \\
\hline
\end{tabular}

a. Equation of the curve relating $I \%$ and $\log C$ (antioxidant concentration). b. Mean \pm standard deviation of two independent experiments performed in triplicate. $\mathrm{IC}_{50}$ values are expressed in $\mathrm{mol} \mathrm{L}^{-1}$.

dimethylsulfoxide. The use of a different reaction medium affects the lifetime and the reactivity of singlet oxygen, which could contribute to the different results.

Moreover, other luminescence methods that describe sodium azide as an ${ }^{1} \mathrm{O}_{2}$ scavenger are based on detection of the near-infrared light emission of ${ }^{1} \mathrm{O}_{2}$, and therefore would not be able to detect the formation of azidyl radical. ${ }^{11,24}$

Mannitol, described as an antioxidant compound with scavenger capacity specific for hydroxyl radical, was tested using concentrations in the range of $1 \times 10^{-5}$ to $1 \times 10^{-2} \mathrm{~mol} \mathrm{~L}^{-1}$. The mannitol concentration had no effect on the analytical signal, which leads us to conclude that there was no scavenging of ${ }^{1} \mathrm{O}_{2}$ and that there was no production of hydroxyl radical.

Reduced glutathione reactivity towards ${ }^{1} \mathrm{O}_{2}$ was also studied; for this purpose, concentrations ranging from $1 \times 10^{-6}$ to $1 \times 10^{-2} \mathrm{~mol} \mathrm{~L}^{-1}$ were tested (Fig. 4). When the assay was performed without $\mathrm{MoO}_{4}{ }^{2-}$ an increase in glutathione concentration resulted in an increase in the CL intensity. The enhancing effect of glutathione in the CL emitted by the reaction of luminol and $\mathrm{H}_{2} \mathrm{O}_{2}$ has been previously reported by Du et al. ${ }^{25}$ This effect was so pronounced that glutathione concentrations higher than $1 \times 10^{-4} \mathrm{~mol} \mathrm{~L}^{-1}$ caused a detector overload, impairing detection. When ${ }^{1} \mathrm{O}_{2}$ was produced, an increase in the CL emission was observed by increasing glutathione concentration. Nevertheless, the analytical signal was lower than the one obtained in the absence of $\mathrm{MoO}_{4}{ }^{2-}$, indicating that glutathione has some scavenging capacity against ${ }^{1} \mathrm{O}_{2}$. However, since not all of the $\mathrm{H}_{2} \mathrm{O}_{2}$ was converted to ${ }^{1} \mathrm{O}_{2}$, the remaining $\mathrm{H}_{2} \mathrm{O}_{2}$ reacted with luminol and glutathione leading to a higher $\mathrm{CL}$ emission, when compared to the $\mathrm{CL}$ emitted when ${ }^{1} \mathrm{O}_{2}$ was produced in the absence of glutathione. Due to the interference of the remaining $\mathrm{H}_{2} \mathrm{O}_{2}$ it was not possible to determine the $\mathrm{IC}_{50}$ of glutathione.

The reactivity values of tryptophan, ascorbic acid and dipyrone, compounds with known antioxidant activity, were also evaluated. During the assay of tryptophan, for lower concentrations a pro-oxidant effect was obtained. By increasing the concentration of tryptophan we observed a decrease in the $\mathrm{CL}$ intensity, indicating that tryptophan has scavenging capacity against ${ }^{1} \mathrm{O}_{2}$. Dipyrone and ascorbic acid also possess scavenging capacity against ${ }^{1} \mathrm{O}_{2}$ (Fig. 5). Inhibition percentages values are presented in Table 1. Ascorbic acid exhibited the highest antioxidant activity, followed by dipyrone and tryptophan.

Nifedipine is an anti-hypertensive drug from the group of calcium channel antagonists. Since reactive oxygen species are involved in several diseases such as atherosclerosis, the potential antioxidant activity of this group of compounds has been the subject of some studies and some scavenging activity against ${ }^{1} \mathrm{O}_{2}$ has been claimed. ${ }^{26}$ Nifedipine concentrations from $1 \times 10^{-5}$ to $1 \times 10^{-3} \mathrm{~mol} \mathrm{~L}^{-1}$ were assayed and it was verified that on increasing nifedipine concentration there was an increase in the CL intensity for both reactions. The mechanism of nifedipine oxidation by superoxide involves the formation of radical products. ${ }^{27}$ A possible explanation for the results obtained in our study is that, as verified for superoxide, nifedipine oxidation by ${ }^{1} \mathrm{O}_{2}$ results in the production of radical species that are able to oxidize luminol.

For the developed flow system, the sampling rate was about 45 determinations per hour with a low sample and reagents consumption per determination: sample, $60 \mu \mathrm{L}$; luminol, $27 \mu \mathrm{g}$; $\mathrm{H}_{2} \mathrm{O}_{2}, 51 \mu \mathrm{g}$; and $\mathrm{Na}_{2} \mathrm{MoO}_{4}, 0.72 \mathrm{mg}$. Multiple analysis of solutions of the distinct compounds showed that the analytical system exhibited a good reproducibility, with relative standard deviation values lower than $4 \%(n=10)$.

\section{Conclusions}

An automated MPFS for in-line generation of ${ }^{1} \mathrm{O}_{2}$ and subsequent chemiluminometric assessment of scavenging capacity against this reactive species was developed.

MPFS advantageous features allowed the implementation of a simple, fast and precise methodology, characterized by a high degree of automation, thus lessening operator intervention and all eventual errors associated with this intervention. The fast reaction zone homogenization, provided by the pulsed nature of the generated flowing stream, enabled the efficient monitoring of the short-lived species, such as the generated ${ }^{1} \mathrm{O}_{2}$, and of the subsequent scavengers activity, under non-equilibrium conditions, which also permitted observers to detect other potentially present short lived intermediates.

In this work, the scavenging effect against ${ }^{1} \mathrm{O}_{2}$ of several compounds was evaluated; unlike what we expected, some of the previously reported ${ }^{1} \mathrm{O}_{2}$ scavengers had an enhancing effect in the CL. The major conclusion of this study is that, depending on the system used as a ROS generator, the detection technique and experimental conditions such as solvents, reaction time and $\mathrm{pH}$, some known antioxidants can behave as pro-oxidants. A set of antioxidants suitable for the detection of a specific ROS by one assessment method might not necessarily be suitable for another one. Therefore, to have a complete profile of an antioxidant one must perform different assays measuring different aspects of the behavior of antioxidants.

\section{Acknowledgements}

The authors would like to acknowledge the financial support given by Fundação para a Ciência e Tecnologia (FCT) under the project PTDC/QUI-QUI/105514/2008. A. F. T. S. thanks FCT for a grant in the ambit of the project BII/REQUIMTE/GABAI9/2008.

\section{References}

1. T. W. Stief, Med. Hypotheses, 2003, 60, 567.

2. S. W. Ryter and R. M. Tyrrell, Free Radical Biol. Med., 
1998, 24, 1520.

3. M. J. Davies, Biochem. Biophys. Res. Commun., 2003, 305, 761.

4. K. C. Das and C. K. Das, Biochem. Biophys. Res. Commun., 2000, 277, 443.

5. I. Kruk, H. Y. Aboul-Enein, T. Michalska, K. Lichszteld, K. Kubasuk-Kladna, and S. Ölgen, Luminescence, 2007, 22, 379.

6. H. Y. Aboul-Enein, I. Kruk, A. Kladna, K. Lichszteld, and T. Michalska, Biopolymers, 2007, 86, 222.

7. N. M. Sachindra, E. Sato, H. Maeda, M. Hosokawa, Y. Niwano, M. Kohno, and K. Miyashita, J. Agric. Food Chem., 2007, 55, 8516.

8. M. Tan, B. Song, G. Wang, and J. Yuan, Free Radical Biol. Med., 2006, 40, 1644.

9. D. Costa, E. Fernandes, J. L. M. Santos, D. C. G. A. Pinto, A. M. S. Silva, and J. L. F. C. Lima, Anal. Bioanal. Chem., 2007, 387, 2071.

10. M. Niedre, M. S. Patterson, and B. C. Wilson, Photochem. Photobiol., 2002, 75, 382.

11. Y. Harada, K. Suzuki, M. Hashimoto, K. Tsukagoshi, and H. Kimoto, Talanta, 2009, 77, 1223.

12. S. Sun, X. Li, G. Zhang, H. Ma, D. Zhang, and Z. Bao, Biochim. Biophys. Acta, 2006, 1760, 440.

13. A. Miyamoto, K. Nakamura, Y. Ohba, N. Kishikawa, K. Nakashima, and N. Kuroda, Anal. Sci., 2006, 22, 73.

14. M. A. J. Rodgers, J. Am. Chem. Soc., 1982, 105, 6201.
15. L. M. Magalhães, M. Lúcio, M. A. Segundo, S. Reis, and J. L. F. C. Lima, Talanta, 2009, 78, 1219.

16. J. L. M. Santos, M. F. T. Ribeiro, A. C. B. Dias, J. L. F. C. Lima, and E. A. G. Zagatto, Anal. Chim. Acta, 2007, 600, 21.

17. K. L. Marques, J. L. M. Santos, and J. L. F. C. Lima, Anal. Lett., 2007, 40, 2241.

18. S. R. P. Meneses, K. L. Marques, C. K. Pires, J. L. M. Santos, E. Fernandes, J. L. F. C. Lima, and E. A. G. Zagatto, Anal. Biochem., 2005, 345, 90.

19. J. M. Aubry and B. Cazin, Inorg. Chem., 1988, 27, 2013.

20. K. Böhme and H. D. Brauer, Inorg. Chem., 1992, 31, 3468.

21. T. Kawatani, J. M. Lin, and M. Yamada, Analyst, 2000, 125, 2075.

22. L. Pecci, M. Costa, G. Montefoschi, A. Antonucci, and D. Cavallini, Biochem. Biophys. Res. Commun., 1999, 254, 661.

23. H. Cui, M. J. Shi, R. Meng, J. Zhou, C. Z. Lai, and X. Q. Lin, Photochem. Photobiol., 2004, 79, 233.

24. K. Tsukagoshi, K. Fukumoto, K. Noda, R. Nakajima, K. Yamashita, and H. Maeda, Anal. Chim. Acta, 2006, 570, 202.

25. J. Du, Y. Li, and J. Lu, Anal. Chim. Acta, 2001, 448, 79.

26. N. A. Pizarro-Urzúa and L. J. Núñez-Vergara, J. Photochem. Photobiol., A, 2005, 175, 129.

27. M. E. Ortiz, L. J. Núñez-Vergara, C. Camargo, and J. A. Squella, Pharm. Res., 2004, 21, 428. 